\title{
Rickettsia raoultii in Haemaphysalis erinacei from marbled polecats, China-Kazakhstan border
}

\author{
Li-Ping Guo ${ }^{1 \dagger}$, Lu-Meng Mu ${ }^{1 \dagger}$, Jun Xur ${ }^{2 \dagger}$, Su-Hua Jiang ${ }^{1 \dagger}$, An-Dong Wang ${ }^{3}$, Chuang-Fu Chen ${ }^{3}$, Gang Guo ${ }^{2}$,
} Wan-Jiang Zhang ${ }^{1}$ and Yuan-Zhi Wang ${ }^{1 *}$

\begin{abstract}
We found Rickettsia raoultii DNA in 2 out of 32 (6.25 \%) Haemaphysalis erinacei ticks. Result showed that the sequences of five genes (17-kDa, gltA, ompA, rrs, and ompB) were $100 \%$ identity with that of R. Raoultii in GenBank. This study is the first report on the presence of $R$. raoultii in $H$. erinacei from wild marbled polecat, Vormela peregusna. Our findings suggest that $H$. erinacei parasitizing wild marbled polecat may serve as reservoir and carriers for $R$. raoultii in areas around the China-Kazakhstan border. The transmission of tick-borne diseases originated from wildlife should not be underestimated in border region.
\end{abstract}

Keywords: Rickettsia raoultii, PCR, Haemaphysalis erinacei, Marbled polecats, China-Kazakhstan border

\section{Findings}

In China, at least five validated spot fever group (SFG) rickettsial species have been detected in ticks, including Rickettsia heilongjiangii, $R$. sibirica [1], $R$, raoultii, $R$. slovaca [2] and $R$. felis [3]. Of these five rickettsial species, none has been identified in the tick Haemaphysalis erinacei. Although no published evidence indicates that $H$. erinacei ticks bites humans, this species is interesting because it coexists with various animal species, including the hedgehog Hemiechinus auritus and the marbled polecat, Vormela peregusna [4], the later is listed as vulnerable globally by the International Union for Conservation of Nature (IUCN) [5]. The marble polecat is distributed from southeast Europe, through southwest and Central Asia, to Mongolia and northern China [6]. In the present study, we determined the presence of $R$. raoultii in $H$. erinacei from marbled polecats in wetlands around Ebinur Lake, northwest China.

Thirty-two adult ticks, 21 (14 male and seven female) from two marbled polecats and 11 (seven male and four female) from three hedgehogs, were collected in wetlands around Ebinur Lake (189 $\mathrm{m}$ above sea level; 82

\footnotetext{
* Correspondence: wangyuanzhi621@126.com

${ }^{\dagger}$ Equal contributors

${ }^{1}$ School of Medicine, Shihezi University, Shihezi 832000, China

Full list of author information is available at the end of the article
}

$48^{\prime} 51 \mathrm{E} 45^{\circ} 04^{\prime} 22 \mathrm{~N}$ ) in northwest China in 2014. The ticks were identified morphologically as $H$. Erinacei and the molecular identification of those ticks by using $16 \mathrm{~S}$ mitochondrial gene results showed that they have a similarity of $90.95 \%$ with that of $H$. concinna (there are no corresponding $16 \mathrm{~S}$ mitochondrial gene sequence for $H$. Erinacei in GenBank). The sequences obtained were deposited in GenBank [GenBank: KR053302-KR053305]. Genomic DNA was extracted from individual specimens by using a TIANamp Genomic DNA Kit (TIANGEN, Beijing, China). A targeting gene fragment (434 bp) from the Rickettsia-specific $17-\mathrm{kDa}$ surface antigen gene was amplified by PCR following a previously published methodology [7]. Another five genetic markers [1332-, 1060-, 488-, 491-, and 812-bp products of the genes encoding 16S rRNA (rrs), citrate synthase ( $g l t A)$, surface cell antigen 1 (sca1), and outer membrane proteins A and $\mathrm{B}(o m p A$ and $o m p B)]$ were amplified by using primers previously described to detect Rickettsia spp. in H. erinacei [8]. The PCR products were sequenced and phylogenetically analyzed to certify the taxonomic identification of the rickettsial agent.

Rickettsial DNA was detected in two (both female) out of $32(6.25 \%) H$. erinacei ticks, which were collected from the same marbled polecat. No rickettsial agent was found in hedgehogs. The sequences BLAST 
results showed that these two rickettsial sequences of five genes $(17-k D a, g l t A, o m p A, r r s$, and $o m p B)$ were the same, and $100 \%$ identity with that of $R$. raoultii. The scal sequences obtained were closest to that of $R$. montanensis str. OSU $85-930$ and $R$. montanensis str. M/5-6, with a sequence similarity of $99.18 \%(612$ out of $617 \mathrm{bp}$ ) (There are no corresponding sequence for $R$. raoultii in GenBank). All of the obtained sequences were deposited in GenBank [GenBank: KR608783KR608788]. The phylogenetic tree produced from the Maximum Likelihood and Neighbor-Joining analyses of the sequence data for the six genes (17-kDa-ompA-gltArrs-sca1-ompB) revealed that the $R$. raoultii obtained from $H$. erinacei was culstered into a clade including " $R$. Raoultii (Heilongjiang, China)", " $R$. raoultii (Russia)", and " $R$. raoultii isolate BL029-2 (Xinjiang, China)" (Fig. 1).

Based on the information in GenBank, $R$. raoultii have been detected at least in 13 tick species, namely: Dermacentor nuttallii, D. marginatus, D. reticulatus, D. silvarum, Rhipicephalus pumilio, $R h$. turanicus, $H$. concinna, H. japonica, Ixodes persulcatus, I. ricinus, Amblyomma helvolum, Hyalomma asiaticum, and $H y$. lusitanicum [9]. However, this study is the first to report the presence of $R$. raoultii in $H$. erinacei. In previous studies, $H$. erinacei has been found in birds, the desert hedgehog Paraechinus aethiopicus, the North African hedgehog Atelerix algirus, stray dogs, the beech marten Martes foina, and the least weasel Mustela nivalis [10-13]. Here our sampling site, the Ebinur Lake, is widely known to be a station for thousands of wildlife around the China-Kazakhstan border. Approximately 1 million migratory birds arrive here, which is known to be home every year, and more than 160 wild vertebrate species and 230 bird species inhabit and/or migrate at this region [14]. Another several previous studies gave the strong evidence that $R$. raoultii is common and widespread across wildlife such as wild snakes, rats and Mongolian gazelle [15-17]. Our findings suggest that $H$. erinacei parasitizing wild marbled polecat may serve as reservoirs and carriers for $R$. raoultii in areas around the China-Kazakhstan border. In the future, the transmission of tick-borne diseases originated from wildlife should not be underestimated in border region. There is a need for international cooperation to survey this and other tick-borne pathogens in migratory birds and wildlife.

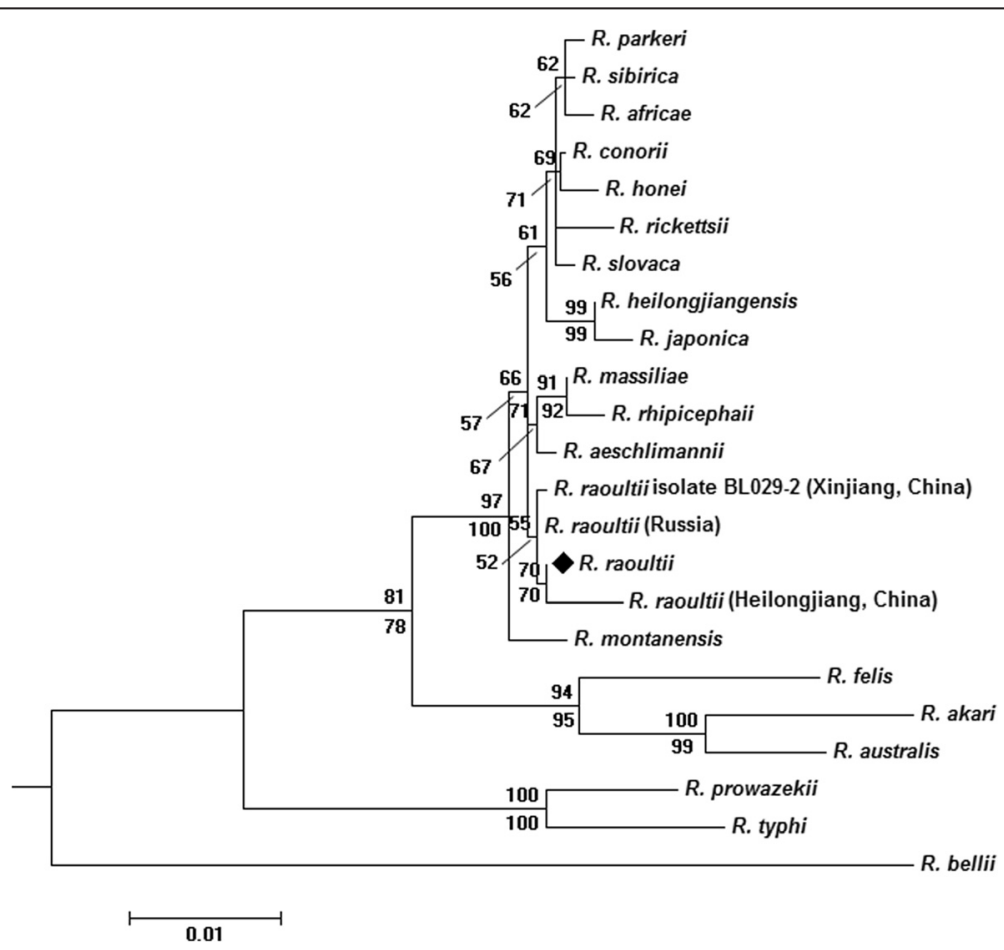

Fig. 1 Phylogenetic tree of 17-kDa-ompA-gltA-rrs-sca1-ompB concatenated sequence of Rickettsia raoultii in Haemaphysalis erinacei $(\bullet)$. The tree was constructed on the basis of Maximum Likelihood (Bootstrap replicates: 1000) and Neighbor-Joining (Bootstrap replicates: 500) analyses of concatenated sequence data of six genes (17-kDa-ompA-gltA-rrs-sca1-ompB) using MEGA6. The sequences of $R$. bellii were used as the outgroup in the concatenated sequence data. The scale bar represents the inferred substitutions per nucleotide site. The relative support for clades in the tree produced from the ML and NJ analyses are indicated above and below branches, respectively 


\section{Competing interests}

The authors declare that they have no competing interests.

\section{Authors' contributions}

YZW conceived the study and participated in its design and coordination. CFC, SHJ, JX, GG and WJZ performed tick collection from the marbled polecats and hedgehogs. ADW and LMM conducted the morphological analyses. LPG, MLM and WAD performed PCR experiments and participated in the sequence alignment. LPG submitted all of the sequences to the GenBank and drafted the manuscript. All authors read and approved the final manuscript.

\section{Acknowledgments}

We thank Chengyao Li for his critical review of the manuscript. This research was supported in part by grants from the National Natural Science Foundation of China (Granted No. 81560338), the National Science \& Technology Pillar Program (No. 2013BAI05B05) and Co-innovation Center for the High Incidence of Zoonotic Disease Prevention and Control in Western China (No. 2013-179).

\section{Author details}

${ }^{1}$ School of Medicine, Shihezi University, Shihezi 832000, China. ${ }^{2}$ Xinjiang Entry-Exit Inspection and Quarantine Bureau, Urumqi 830063, China. ${ }^{3}$ College of Animal Science and Technology, Shihezi University, Shihezi 832000, China.

Received: 15 July 2015 Accepted: 1 September 2015

Published online: 17 September 2015

\section{References}

1. Zhang JZ, Fan MY, Wu YM, Fournier PE, Roux V, Raoult D. Genetic classification of "Rickettsia heilongjiangii" and "Rickettsia hulinii", two Chinese spotted fever group Rickettsiae. J Clin Microbiol. 2000;38(9):3498-501.

2. Tian ZC, Liu GY, Shen H, Xie JR, Luo J, Tian MY. First report on the occurrence of Rickettsia slovaca and Rickettsia raoultii in Dermacentor silvarum in China. Parasit Vectors. 2012;5:19.

3. Zhang J, Lu G, Kelly P, Zhang Z, Wei L, Yu D, et al. First report of Rickettsia felis in China. BMC Infect Dis. 2014;14:682.

4. Yin XP, Ye ZH, Jiao W, Ma DH, Zhang JG, Abudu Z. Tick fauna and monitoring of tick host animals at Alataw Pass. Chin J Vector Biol Control. 2010;21(4):375-7.

5. IUCN 2008. 2008 IUCN Red List of Threatened Species. IUCN. http://www.iucnredlist.org/details/29680/0. Accessed 30 Jun 2008

6. Ibiş $\mathrm{O}, \mathrm{Tez} \mathrm{C}$. Phylogenetic status and genetic diversity of the Turkish marbled polecat, Vormela peregusna, (Mustelidae: Carnivora: Mammalia), inferred from the mitochondrial cytochrome $b$ gene. Vert Zoonol. 2014;62(4):285-94.

7. Anstead CA, Chilton NB. Detection of a novel Rickettsia (Alphaproteobacteria: Rickettsiales) in rotund ticks (Ixodes kingi) from Saskatchewan, Canada. Ticks Tick-Borne Dis. 2013;4(3):202-6.

8. Anstead CA, Chilton NB. A novel Rickettsia species detected in vole ticks (Ixodes angustus) from Western Canada. Appl Environ Microbiol. 2013;79(24):7583-9.

9. Wen J, Jiao D, Wang JH, Yao DH, Liu ZX, Zhao G, et al. Rickettsia raoultii, the predominant Rickettsia found in Dermacentor silvarum ticks in China-Russia border areas. Exp Appl Acarol. 2014;63(4):579-85.

10. Tovornik D, Cerný V. Finding of Haemaphysalis erinacei erinacei Pavesi, 1884 on birds in Yugoslavia. Folia Parasitol (Praha). 1974;21(3):282.

11. Hosseini-Chegeni A, Arzamani K, Banafshi O, Telmadarraiy Z, Salimi M. A record of Haemaphysalis erinacei (Acari: Ixodidae) collected from hedgehog and an identification key for the species of Haemaphysalis occurring in Iran. Pers J Acarol. 2014;3(3):203-15.

12. Qablan MA, Kubelová M, Siroký P, Modrý D, Amr ZS. Stray dogs of northern Jordan as reservoirs of ticks and tick-borne hemopathogens. Parasitol Res. 2012;111(1):301-7.

13. Lorusso V, Lia RP, Dantas-Torres F, Mallia E, Ravagnan S, Capelli G, et al Ixodid ticks of road-killed wildlife species in southern Italy: new tick-host associations and locality records. Exp Appl Acarol. 2011;55(3):293-300.

14. Ma M, Bayahen K, Li F, Hu BW, Wu JQ, Douglas M, et al. List of birds and count of autumn migration in Ebinur Wetland Nature Reserve. Sichuan Journal of Zoology. 2010;29(6):912-8.
15. Kho KL, Koh FX, Tay ST. Molecular evidence of potential novel spotted fever group rickettsiae, Anaplasma and Ehrlichia species in Amblyomma ticks parasitizing wild snakes. Parasit Vectors. 2015;8:112.

16. Tay ST, Mokhtar AS, Low KC, Mohd Zain SN, Jeffery J, Abdul Aziz N, et al. Identification of rickettsiae from wild rats and cat fleas in Malaysia. Med Vet Entomol. 2014;28 Suppl 1:104-8.

17. Li Y, Chen Z, Liu Z, Liu J, Yang J, Li Q, et al. First report of Theileria and Anaplasma in the Mongolian gazelle, Procapra gutturosa. Parasit Vectors. 2014;7:614.

\section{Submit your next manuscript to BioMed Central and take full advantage of:}

- Convenient online submission

- Thorough peer review

- No space constraints or color figure charges

- Immediate publication on acceptance

- Inclusion in PubMed, CAS, Scopus and Google Scholar

- Research which is freely available for redistribution 